\title{
The performance of shear wave elastography on evaluating liver changes in obese and overweight children
}

\author{
Neslihan Özkul Sağlam ${ }^{1 \oplus,}$, Sema Aksoy ${ }^{2 \oplus}$, Selcen Yaroğlu Kazanc1 ${ }^{1 \oplus}$, \\ Figen Palabıyık ${ }^{2 \oplus}$, Sadık Sami Hatipoğlu ${ }^{1 \oplus}$, Ercan İnci ${ }^{2 \oplus}$ \\ ${ }^{1}$ Division of General Pediatrics, Department of Pediatrics and ${ }^{2}$ Department of Radiology, Bakirkoy Dr Sadi Konuk Training and \\ Research Hospital, Istanbul, Turkey.
}

\begin{abstract}
Background. Real time shear-wave elastography (SWE) is a non-invasive imaging method which can quantitatively assess liver stiffness. Obesity and its complication are increasing with improving lifestyles in our century. We evaluated the performance of SWE for detecting liver changes (fatty liver, steatohepatitis) in obese and overweight children, in addition to this, we determined the diagnostic accuracy and clinical usefulness of SWE in non-alcoholic fatty liver disease (NAFLD).

Methods. Obese and overweight 41 children within the age range of 6-15 years were included in this singlecenter prospective study. Biochemical evaluation for aspartate aminotransferase (AST), alanine aminotransferase (ALT), triglyceride (TG), total cholesterol (TC) levels, as well as conventional ultrasound and SWE of the liver were performed in the patient group. These values were compared with values of 25 normal weight and healthy children in the age range of $6-16$ years.

Results. The mean SWE values was $13.7 \pm 5.5 \mathrm{kiloPascal}(\mathrm{kPa})$ and $2.03 \pm 0.35$ meter/second $(\mathrm{m} / \mathrm{s})$ in patient group and $7.99 \pm 2.81 \mathrm{kPa}$ and $1.62 \pm 0.21 \mathrm{~m} / \mathrm{s}$ in control group $(\mathrm{p}<0.01)$. The receiver-operating characteristics (ROC) analysis was performed to determine the optimum cut-off value for elastography values ( $\mathrm{kPa}$ ) to evaluate liver changes; area under the curve was $87.5 \%$ (95\% CI 79.3-95.8). When the cutoff value was set as $10.45 \mathrm{kPa}$, the sensitivity and the specificity was $69.2 \%$ and $100 \%$, respectively. We could not observe a statistically significant difference when we compared the elastography values $(\mathrm{kPa}$ and $\mathrm{m} / \mathrm{s})$ according to presence of hepatosteatosis $(\mathrm{p}=0.581$ and 0.172 ). There were no significant correlations between SWE and AST, ALT values.
\end{abstract}

Conclusions. SWE may be a useful and accurate imaging method to evaluate liver changes and monitor NAFLD in obese and overweight children.

Key words: child, obesity, liver, real time shear-wave elastography.

The worldwide prevalence of childhood obesity has increased constantly during the past three decades according to a report from the World Health Organization in 2011.1,2 The population of overweight children and adolescents under 18 years of age is 17 million with an annual increase of $0.5-1$ percent. ${ }^{3}$ However, this rise has caused an increased incidence of obesity comorbidities, including hyperlipidemia and non-alcoholic

$凶$ Neslihan Özkul Sağlam

neslisaglam73@gmail.com

Received 9th May 2018, revised 26th April 2019, accepted 25th May 2020. fatty liver disease (NAFLD). ${ }^{4}$ NAFLD is the most common pediatric liver disease and its prevalence has more than doubled in the last decades $^{5} ; 3-11 \%$ of the pediatric population present NAFLD $^{6,7}$ with highest prevalence (46\%) reached among overweight and obese children and adolescents. ${ }^{8}$

NAFLD includes conditions changing from fatty liver or steatohepatitis to cirrhosis and its complications (e.g. hepatocellular carcinoma and portal hypertension). ${ }^{9}$ Although there are some noninvasive diagnostic tools for NAFLD, liver biopsy is the gold standard for diagnosis. It gives to the clinicians the possibility to both 
diagnose the NAFLD and assess its progression to fibrosis or cirrhosis with greater certainty than other techniques. However, the use of liver biopsy in routine clinical practice has some drawbacks. Most cases with NAFLD have good prognosis, for this reason, the risks of a liver biopsy appear to overweigh the clinical benefits. It represents an impractical screening procedure as it is invasive, expensive and has complications such as pain or hemorrhage. Furthermore, inaccurate sampling of liver biopsy can cause very serious misdiagnosis as well as staging inaccuracies, because histological lesions of non-alcoholic steatohepatitis (NASH) have an uneven distribution in liver parenchyma. As the liver biopsy is limited, clinicians have to use noninvasive and practical methods to diagnose and stage NAFLD. ${ }^{9}$

Recently, some noninvasive methods for measuring liver stiffness (LS), including transient elastography (TE), acoustic radiation force impulse imaging (ARFI), and magnetic resonance elastography have been developed. ${ }^{10}$ More recently, real-time shear wave elastography (SWE), another method for measuring LS, has been developed. ${ }^{11}$

SWE is a novel noninvasive method that involves application of local mechanical compression on soft tissue using focused ultrasound scanning (US) and acquiring strain images that show tissue responses. ${ }^{12,13}$ Several studies report positive results in their ability to predict the degree of hepatic fibrosis. ${ }^{14-16}$ Unlike TE, SWE measures tissue elasticity simultaneously during B-mode ultrasound examination, and elasticity values can be measured on the basis of anatomical information. In addition, SWE provides elastography color, quantitative maps according to the degree of stiffness, allowing an assessment of homogeneity. ${ }^{10}$ The spatial heterogeneity of liver stiffness can be visualized, and the size of the region used for a measurement can be selectively placed or adjusted. SWE is a relatively new method, therefore studies in children are limited. ${ }^{17}$

The aim of this study was to evaluate the performance of real time SWE for detecting liver changes (fatty liver or steatohepatitis) in obese and owerweight children, in addition to this, to determine the diagnostic accuracy and clinical usefulness of SWE in NAFLD in the absence of liver biopsy.

\section{Material and Methods}

\section{Study population}

This was a single center, cross-sectional and prospective study. The patient group included 41 children in the age range of 6-15 years who were admitted to University of Health Sciences Bakırkoy Dr. Sadi Konuk Training and Research Hospital, general pediatrics outpatient clinic between June 2015 and January 2016.

The study protocol was approved by the University of Health Sciences Bakırkoy Dr. Sadi Konuk Training and Research Hospital Ethics Committee (07.12.2015, report number: 2015/19/01). All subjects gave signed informed consent for the research. Inclusion criteria were the presence of obesity or being overweight. Patients who had a body mass index (BMI) at or above the 95th percentile (obesity) and at or above the 85th percentile and below the 95th percentile (overweight) were included in this study. Children with known chronic disease and liver disease were excluded.

Demographic data were noted. The body weight and height were measured for all patients and BMI was calculated. To define obesity, the percentile distributions relative to gender and age which were constituted by Neyzi et al. (2015 Growth Charts) were used..$^{18}$ All of the patients underwent a physical examination. We evaluated laboratory tests, which included alanine aminotransferase (ALT), aspartate aminotransferase (AST), triglyceride (TG) and total cholesterol (TC). The values below 35 $\mathrm{U} / \mathrm{L}$ for both AST and ALT was considered as normal. The normal cutoff value for TG level was $150 \mathrm{mg} / \mathrm{dl}$ and for TC level was $200 \mathrm{mg} / \mathrm{dl}$. Conventional ultrasound and real time SWE for each patient were performed. All ultrasounds 
were performed by a single observer (SA). All clinical details, examinations, laboratory and radiological findings were recorded.

The control group included 25 healthy children that were admitted to our outpatient clinic, within the age range of 6-16 years, whose BMI were between the 5th percentile and 85th percentile. These children showed a normal physical examination, normal levels of transaminase and lipids. Their liver ultrasound revealed no change by conventional methods. SWE was also performed on the children in the control group.

\section{Real time shear-wave elastography}

Measurements were performed using convex probe on an Applio 500 Platinum device (Toshiba, America, medical Systems) by the same radiologist. The patients were examined lying in dorsal decubitus position with the right arm at maximal abduction in a breath suspension mid-respiratory phase.

The region of interest (ROI) $(10 \times 16 \mathrm{~mm})$ was put in the right lobe of the liver. Measurements were performed 1.5 to $2.0 \mathrm{~cm}$ below the liver capsule through intercostal spaces in the right lobe. The area was far from the blood vessels that could be caused by artifacts. The values were reported as shear wave speed $(\mathrm{m} / \mathrm{s})$ and converted to $\mathrm{kPa}$ (kiloPascal; elastic modulus).

\section{Statistical analysis}

Statistical analysis was performed by using IBM SPSS (Statistics Package for Social Sciences for Windows, Version 21.0, Armonk, NY, IBM Corp.) package program. The chi-square test was used to determine the differences between categorical variables. To show the behavioral differences of group averages; analysis of variance (ANOVA) T-test was used when normality and uniformity variables were met, and the statistical analyses for continuous variables were performed using the MannWhitney $U$ test. In addition, while comparing the value of any parameter between the groups, analysis of covariance (ANCOVA) analysis was performed to determine a statistically significant difference, taking into account the factors affecting this change. In calculating the correlation between any two numerical variables, the nonparametric Spearman's rank correlation test was used because the data did not have a normal distribution. The receiveroperating characteristics (ROC) analysis was performed to test optimum cutoff value for elastography values $(\mathrm{kPa})$ to evaluate liver changes (fatty liver or steatohepatitis). Statistical significance for all cases was determined as $\mathrm{p}$ $<0.05$.

\section{Results}

Demographics, clinical features and laboratory findings of the patient and control groups are summarized in Table I. Seven patients (17.1\%) had hepatosteatosis (mild) on conventional US. Out of 41 obese and overweight children, AST was found to be raised in 1 child $(2.4 \%)$ and ALT was raised in 3 children (7.3\%). Only nine $(21.9 \%)$ patients had TG levels of $>150 \mathrm{mg} / \mathrm{dl}$. None of the healthy children had elevated levels of TG. Only two $(4.8 \%)$ patients had TC levels of $>200 \mathrm{mg} / \mathrm{dL}$. One of the healthy children had elevated level of TC.

SWE values were higher in patients, compared to controls ( $p<0.001$, Table II).

The receiver-operating characteristics (ROC) analysis to determine the optimum cutoff value for elastography values $(\mathrm{kPa})$ to evaluate liver changes revealed an area under the curve of $87.5 \%$ (95\% CI 79.3 - 95.8). When the cutoff value was set as $10.45 \mathrm{kPa}$, the sensitivity and the specificity was $69.2 \%$ and $100 \%$, respectively (Fig 1).

The elastography values ( $\mathrm{kPa}$ and $\mathrm{m} / \mathrm{s}$ ) were positively correlated with weight, BMI, BMISDS, TG values and presence of acanthosis; there was no correlation between elastography values and age, height, ALT, AST, TC (Table III).

When the patient and control group were compared in terms of elastography values with 
Table I. Demographics, clinical features and laboratory findings of the patient and control groups.

\begin{tabular}{lllc}
\hline Characteristics & $\begin{array}{l}\text { Patient Group } \\
(\mathrm{N}=41)\end{array}$ & $\begin{array}{l}\text { Control Group } \\
(\mathrm{N}=25)\end{array}$ & p-value \\
\hline Age $($ year $)$ & $11.39(9.6,13.53)$ & $11.7(9,13.2)$ & $0.905^{*}$ \\
Sex $($ female/male), $\mathrm{n} / \mathrm{n}$ & $19 / 22$ & $13 / 12$ & $0.847^{* *}$ \\
BMI $\left(\mathrm{kg} / \mathrm{m}^{2}\right)$ & $27.87(25.08,31.5)$ & $18.66(17.2,20.7)$ & $<0.001^{*}$ \\
BMI-SDS & $2.31(2,2.79)$ & $0.36(-0.17,0.86)$ & $<0.001^{*}$ \\
Acanthosis, $\mathrm{n}(\%)$ & $19(46.3)$ & 0 & $<0.001^{* *}$ \\
ALT $(\mathrm{IU} / \mathrm{L})$ & $18(15,22)$ & $13(10.07,16.25)$ & $0.001^{*}$ \\
AST $(\mathrm{IU} / \mathrm{L})$ & $21(19,23)$ & $23(17.75,25.25)$ & $0.569^{*}$ \\
Total cholesterol $(\mathrm{mg} / \mathrm{dl})$ & $156(142,172.75)$ & $137.5(128,149.75)$ & $0.074^{*}$ \\
Triglyceride $(\mathrm{mg} / \mathrm{dl})$ & $107.5(87,135.5)$ & $68(59.25,98.25)$ & $0.014^{*}$ \\
Hepatosteatosis, $\mathrm{n}(\%)$ & $7(17.1)$ & 0 & $0.039^{* *}$ \\
Elastography $(\mathrm{kPa})$ & $13(8.9,16.9)$ & $7.4(6.8,8.5)$ & $<0.001^{*}$ \\
Elastography $(\mathrm{m} / \mathrm{s})$ & $2.07(1.8,2.21)$ & $1.59(1.52,1.73)$ & $<0.001^{*}$ \\
\hline
\end{tabular}

Data are presented as median $(\mathrm{Q} 1, \mathrm{Q} 3)$ or $\mathrm{n}(\%)$.

*: Mann-Whitney U test; **: Chi-square test

ALT: alanine transaminase, AST: aspartate transaminase, BMI: body mass index, BMI-SDS: body mass index-standard deviation score.

Table II. The mean SWE values of patients and controls.

\begin{tabular}{lccl}
\hline \multirow{2}{*}{ SWE values } & \multicolumn{2}{c}{ Groups } & \multirow{2}{*}{ p-value } \\
\cline { 2 - 3 } & Patients $(\mathrm{N}=41)$ & Controls $(\mathrm{N}=25)$ & $<0.001^{*}$ \\
\hline Elastography $(\mathrm{kPa})$ & $13(8.9,16.9)$ & $7.4(6.8,8.5)$ & $<0.001^{*}$ \\
Elastography $(\mathrm{m} / \mathrm{s})$ & $2.07(1.8,2.21)$ & $1.59(1.52,1.73)$ & \\
\hline
\end{tabular}

Data are presented as median (Q1, Q3).

*: Mann-Whitney U test

Table III. Correlation analysis between elastography and certain clinical and laboratory parameters.

\begin{tabular}{lcccc}
\hline Parameters & \multicolumn{2}{c}{ Elastography $(\mathrm{kPA})$} & \multicolumn{2}{c}{ Elastography $(\mathrm{m} / \mathrm{s})$} \\
\hline Age & $\mathrm{r}^{*}$ & $\mathrm{p}$ & $\mathrm{r}^{*}$ & $\mathrm{p}$ \\
Height & 0.209 & 0.092 & 0.202 & 0.135 \\
Weight & 0.21 & 0.091 & 0.167 & 0.219 \\
ALT & 0.559 & $<0.001$ & 0.537 & $<0.001$ \\
AST & 0.255 & 0.058 & 0.283 & 0.051 \\
BMI & -0.091 & 0.488 & -0.069 & 0.624 \\
BMI-SDS & 0.705 & $<0.001$ & 0.688 & $<0.001$ \\
Total cholesterol & 0.665 & $<0.001$ & 0.641 & $<0.001$ \\
Triglyceride & 0.239 & 0.127 & 0.305 & 0.066 \\
Acantosis & 0.443 & 0.002 & 0.444 & 0.005 \\
\hline
\end{tabular}

*: Spearman's correlation test

ALT: alanine transaminase, AST: aspartate transaminase, BMI: body mass index, BMI-SDS: body mass index-standard deviation score. 


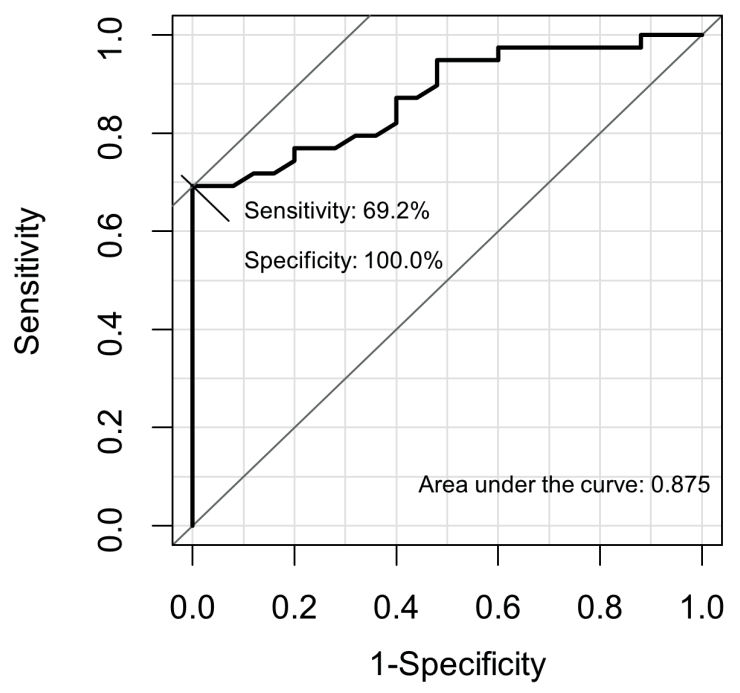

Fig. 1. The receiver-operating characteristics (ROC) analysis was performed to determine the optimum cutoff value for elastography values $(\mathrm{kPa})$ to detect liver changes in obese and overweight children; area under the curve was $87.5 \%$ (95\% CI 79.3 - 95.8). When the cutoff value was set as $10.45 \mathrm{kPa}$, the sensitivity and the specificity was $69.2 \%$ and $100 \%$, respectively.

ANCOVA, we found that AST, ALT, TC did not affect these results ( $p$ values $0.373,0.882$, 0.143 , respectively). We determined the effect of BMI, BMI-SDS and TG on elastography values (all $\mathrm{p}$ values $<0.001$ ). SWE values were higher in the presence of acanthosis (13.15 \pm 1.505 vs. $8.89 \pm 1.99 \mathrm{kPa} ; \mathrm{p}=0.002)$. SWE values were comparable between groups with and without hepatosteatosis (Table IV).

\section{Discussion}

The initial step to determine the potential risk for obesity and NAFLD requires that the primary care pediatricians calculate and record the BMI for each child at each visit. NAFLD is usually "a silent liver disease" because it occurs without any warning signs. Some symptoms of chronic liver disease will occur when NASH develops with more severe liver damage. In clinical practice, NAFLD is generally suspected based on the findings of hypertransaminasemia and/or an ultrasonographic bright liver in an otherwise healthy child who is overweight or obese. $^{19}$

Although there are some diagnostic techniques to evaluate NAFLD, liver biopsy is the gold standard for diagnosis. As liver biopsy is an invasive method and most patients with NAFLD have a good prognosis, this method causes controversy and this cannot be performed routinely in the pediatric population. ${ }^{9}$

Conventional US is often the first imaging modality used to evaluate fatty liver clinically, especially for screening of suspected NAFLD, due to its lack of invasiveness, wide availability, and relatively low cost. ${ }^{20,21}$ However, in two studies that have compared liver conventional US and liver histology for measuring the degree of hepatic steatosis, they concluded that conventional US is an insensitive technique to identify mild steatosis. ${ }^{22,23}$ Steatosis is reported to be detectable by US when more than $20 \%$ of hepatocytes contain histologically visible fat droplets, with a reported sensitivity of $79.7 \%$ and specificity of $86.2 \%{ }^{24}$

There are several limitations of conventional US for NAFLD evaluation: 1) It is qualitative and therefore subjective; the value of conventional US to evaluate NAFLD is limited by the subjective nature of the criteria used to differentiate fatty from normal liver and a lack of sonographic criteria for different degrees of

Table IV. The elastography values according to the condition of hepatosteatosis.

\begin{tabular}{lccc}
\hline \multirow{2}{*}{ SWE values } & \multicolumn{2}{c}{ Groups } & \multirow{2}{*}{ p-value } \\
\cline { 2 - 3 } & With hepatosteatosis $(\mathrm{N}=7)$ & Without hepatosteatosis $(\mathrm{N}=59)$ & $0.581^{*}$ \\
\hline Elastography $(\mathrm{kPa})$ & $9.2(7.7,17.3)$ & $9.1(7.52,14.35)$ & $0.172^{*}$ \\
Elastography $(\mathrm{m} / \mathrm{s})$ & $2.17(1.7,2.34)$ & $1.74(1.56,2.07)$ & \\
\hline
\end{tabular}

Data are presented as median (Q1, Q3).

*: Mann-Whitney U test 
steatosis. 2) Sensitivity is limited when there are few steatotic hepatocytes. ${ }^{24}$ 3) The sensitivity and specificity of B mode sonography decreases as BMI increases, varying between $49 \%-100 \%$ and $75 \%-95 \%{ }^{25}$ Conventional US cannot differentiate steatosis and steatohepatitis or stage fibrosis. ${ }^{26}$

SWE quantitatively evaluates liver stiffness to make noninvasive evaluation of liver fibrosis and NASH clinically possible. Estimated tissue stiffness therefore provides information on the presence and degree of fibrosis. ${ }^{27}$

Our study was an effort to diagnose liver changes at its earliest stage in overweight and obese children with fatty liver disease using the new US technique of SWE. It was aimed to determine the accuracy of this technique in the follow-up of NAFLD findings in obese and overweight children.

Different ultrasound-based elastography techniques have been developed to noninvasively evaluate tissue stiffness like that of the liver. The radiologic imaging methods can estimate liver stiffness as a surrogate for liver fibrosis, including SWE. There are many reports related to these methods in adults and children. Guzmán-Aroca et al. ${ }^{28}$ assessed the liver findings in 23 morbid obese adults with acustic radiation force impulse elastography (ARFI) and conventional ultrasound. They showed that the findings of the liver biopsy were correlated with ARFI measurements and considered the ARFI technique as a useful diagnostic tool for differentiating NAFLD from $\mathrm{NASH}$ in asymptomatic patients with morbid obesity.

Real-time SWE is a newer technic and there are limited studies in children with SWE. Tutar et al. ${ }^{17}$ evaluated the liver stiffness with SWE in pediatric chronic liver disease. Consequently, they determined correlation with SWE measurements and fibrosis in liver. Kim et al. ${ }^{29}$ evaluated the diagnostic performance of SWE for determining the severity of liver fibrosis in children and adolescents. They concluded that
SWE is an excellent method for the assessment of the severity of liver fibrosis in children and adolescents.

In our study we compared the liver SWE measurements in obese and overweight children with a healthy control group. The values of the study group were significantly higher than the values of the control group ( $\mathrm{p}<0.001$ for $\mathrm{kPa}$ ). This result has shown that real-time SWE may be used for monitoring liver changes in obese and overweight children.

There are few studies with ultrasound-based elastography that evaluate the liver changes in obese and overweight children. Kamble et al. ${ }^{30}$ have analyzed the diagnostic effectiveness of ARFI elastography with biochemical markers for evaluating hepatic changes in overweight and obese children. In this study ARFI elastography has shown excellent correlation with AST/ALT ratios in obese children. They have concluded that ARFI elastography can be used as a noninvasive method to detect NAFLD and associated hepatic changes in pediatric patients. Marginean et al. ${ }^{31}$ determined that in the group with hepatic steatosis, the SWE values were statistically higher compared to those in healthy controls. They established positive statistical correlations between AST and SWE in the group of children with NAFLD.

As far as we know, there is only one report which evaluated the alterations in liver with SWE in overweight and obese children. Bailey at al. ${ }^{32}$ evaluated US-SWE velocities of the liver in normal-weight and obese children, to correlate US-SWE findings with age and BMI, and to compare US-SWE values with qualitative assessment of the liver by conventional US. It was concluded that US-SWE provides a useful quantitative imaging biomarker for evaluating liver stiffness in children.

Our results showed that the liver SWE values of obese and overweight children were significantly higher than the values of the healthy control group. The calculated area under the ROC curve values indicated a good 
performance of SWE for evaluating the liver changes (fatty liver) in obese and overweight children.

NAFLD is the most common cause of hypertransaminasemia in children and adolescents. $^{33}$ However elevated ALT is not a sensitive marker of disease existence and/ or severity at ordinarily used thresholds. ${ }^{34}$ According to the Screening ALT for Elevation in Today's Youth Study, normal values of transaminases for teenagers and children are presently set too high to detect liver steatosis. ${ }^{35}$ In our study ALT levels of the patient group were significantly higher than the control group. Nevertheless, ALT, AST levels and elastography values were not significantly correlated. Therefore, we think that the serum aminotransferase levels are not reliable biomarkers to detect the degree of fatty liver in obese and overweight children.

Imaging methods, such as conventional US have been increasingly approved as noninvasive alternative methods to diagnose and monitor NAFLD/NASH. US is safe, but it is limited by the inability to detect fatty liver (liver brightness vs. kidney parenchymal echogenicity) when steatosis involves $<30 \%$ of hepatocytes. ${ }^{19}$ Wong et al. ${ }^{36}$ reported that hepatic steatosis necroinflammation, and obesity did not affect the liver stiffness measurement. In our study when the elastography values $(\mathrm{kPa})$ of the group who had hepatosteatosis were compared with those who had no hepatosteatosis, no significant difference was observed. However, the number of patients with hepatosteatosis was very low and all of them were mild hepatosteatosis. It is possible that mild hepatosteatosis may not change the value of SWE. It was considered that further studies are needed on a large number of pediatric populations to evaluate this condition.

Kamble et al. ${ }^{30}$ reported an association between liver changes and TG levels in children. In our study, we found a significant correlation between elastography $(\mathrm{kPa}, \mathrm{m} / \mathrm{s})$ and TG values $(p<0.001)$. We think that SWE and TG level may be useful indicators in the follow-up of NAFLD due to obesity.

In several studies, normal mean liver elasticity values in children were reported. On transient elastographic (Fibro Scan) studies, normal mean liver elasticity values were reported to range between 4.4 and $5.6 \mathrm{kPa} \cdot{ }^{37}$ In the study by Hanquinet et al. ${ }^{38}$ normal mean liver elasticity was reported to be $1.12 \mathrm{~m} / \mathrm{s}$ in healthy children. Tutar et al. ${ }^{17}$ they found the mean elasticity of $7.41 \mathrm{kPa}$ and $1.56 \mathrm{~m} / \mathrm{s}$ in the liver of normal healthy children. In our study, we determined that the mean elasticity of the liver was $7.99 \pm 2.81$ $\mathrm{kPa}$ and $1.62 \pm 0.21 \mathrm{~m} / \mathrm{s}$ in healthy children.

There were some limitations in our study. We could not compare SWE measurements of the liver with the histological results of liver biopsy. This was not possible because liver biopsy is an invasive method. Secondly, measurement of the liver stiffness with SWE were made by the same operator at one time and only in one period; it is difficult to perform this method in children and families did not want to get another imaging.

In conclusion, SWE may a useful and accurate imaging method to evaluate and monitor NAFLD-related liver changes in obese children. However further longitudinal studies are required on a larger pediatric population.

\section{Author contribution}

The authors confirm contribution to the paper as follows: study conception and design: NÖS, SA, FBP, SSH, Eİ; data collection: NÖS; analysis and interpretation of results: NÖS, SYK; draft manuscript preparation: NÖS. All authors reviewed the results and approved the final version of the manuscript.

\section{Ethical approval}

The study protocol was approved by the University of Health Sciences Bakırkoy Dr. Sadi Konuk Training and Research Hospital Ethics Committee (07.12.2015, report number: 2015/19/01). 


\section{Source of funding}

No external funding was received for this study.

\section{Conflict of interest}

The authors have indicated they have no potential conflicts of interest to disclose.

\section{REFERENCES}

1. World Health Organization. Global status report on noncommunicable diseases 2010. Geneva: World Health Organization, 2011: 22-24.

2. Marzuillo P, Grandone A, Conte M, et al. Novel association between a nonsynonymous variant $(\mathrm{R} 270 \mathrm{H})$ of the G-protein-coupled receptor 120 and liver injury in children and adolescents with obesity. J Pediatr Gastroenterol Nutr 2014; 59: 472-475.

3. Lobstein $\mathrm{T}$, Baur L, Uauy R, IASO International Obesity Task Force. Obesity in children and young people: a crisis in public health. Obes Rev 2004; 5(Suppl 1): 4-104.

4. Marzuillo P, Del Giudice EM, Santoro N. Pediatric non-alcoholic fatty liver disease: new insights and future directions. World J Hepatol 2014; 6: 217-225.

5. Marzuillo P, Miraglia del Giudice E, Santoro N. Pediatric fatty liver disease: role of ethnicity and genetics. World J Gastroenterol 2014; 20: 7347-7355.

6. Welsh JA, Karpen S, Vos MB. Increasing prevalence of nonalcoholic fatty liver disease among United States adolescents, 1988-1994 to 2007-2010. J Pediatr 2013; 162: 496-500.e1.

7. Giorgio V, Prono F, Graziano F, Nobili V. Pediatric non alcoholic fatty liver disease: old and new concepts on development, progression, metabolic insight and potential treatment targets. BMC Pediatr 2013; 13: 40.

8. Schwimmer JB, Deutsch R, Kahen T, Lavine JE, Stanley C, Behling C. Prevalence of fatty liver in children and adolescents. Pediatrics 2006; 118: 13881393.

9. Marzuillo P, Grandone A, Perrone L, Miraglia Del Giudice E. Controversy in the diagnosis of pediatric non-alcoholic fatty liver disease. World J Gastroenterol 2015; 21: 6444-6450.

10. Jeong JY, Kim TY, Sohn JH, et al. Real time shear wave elastography in chronic liver diseases: accuracy for predicting liver fibrosis, in comparison with serum markers. World J Gastroenterol 2014; 20: 13920-13929.
11. Muller M, Gennisson JL, Deffieux T, Tanter M, Fink M. Quantitative viscoelasticity mapping of human liver using supersonic shear imaging: preliminary in vivo feasibility study. Ultrasound Med Biol 2009; 35: 219-229.

12. Mauldin FW Jr, Zhu HT, Behler RH, Nichols TC, Gallippi CM. Robust principal component analysis and clustering methods for automated classification of tissue response to ARFI excitation. Ultrasound Med Biol 2008; 34: 309-325.

13. Boursier J, Isselin G, Fouchard-Hubert I, et al. Acoustic radiation force impulse: a new ultrasonographic technology for the widespread noninvasive diagnosis of liver fibrosis. Eur J Gastroenterol Hepatol 2010; 22: 1074-1084.

14. Castéra L, Vergniol J, Foucher J, et al. Prospective comparison of transient elastography, Fibrotest, APRI and liver biopsy for the assessment of fibrosis in chronic hepatitis C. Gastroenterology 2005; 128: 343-350.

15. Friedrich-Rust M, Nierhoff J, Lupsor $M$, et al Performance of acoustic radiation force impulse imaging for the staging of liver fibrosis: a pooled meta-analysis. J Viral Hepat 2012; 19: e212-e219.

16. Huwart L, Sempoux C, Vicaut E, et al. Magnetic resonance elastography for the noninvasive staging of liver fibrosis. Gastroenterology 2008; 135: 32-40.

17. Tutar O, Beşer ÖF, Adaletli I, et al. Shear wave elastography in the evaluation of liver fibrosis in children. J Pediatr Gastroenterol Nutr 2014; 58: 750755.

18. Neyzi O, Bundak R, Gökçay G, Günöz H, Furman A, Darendeliler F, Baş F. Reference values for weight, height, head circumference, and body mass index in Turkish Children. J Clin Res Pediatr Endocrinol 2015; 7: 280-293.

19. Clemente MG, Mandato C, Poeta M, Vajro P. Pediatric non-alcoholic fatty liver disease: recent solutions, unresolved issues, and future research directions. World J Gastroenterol 2016; 22: 80788093.

20. Chen CL, Cheng YF, Yu CY, et al. Living donor liver transplantation: the Asian perspective. Transplantation 2014; 97 (Suppl 8): S3.

21. Singh D, Das CJ, Baruah MP. Imaging of nonalcoholic fatty liver disease: A road less travelled. Indian J Endocrinol Metab 2013; 17: 990-995.

22. Joseph AE, Saverymuttu SH, Al-Sam S, Cook MG, Maxwell JD. Comparison of liver histology with ultrasonography in assessing diffuse parenchymal liver disease. Clin Radiol 1991; 43: 26-31. 
23. Saverymuttu SH, Joseph AE, Maxwell JD. Ultrasound scanning in the detection of hepatic fibrosis and steatosis. Br Med J (Clin Res Ed) 1986; 292: 13-15.

24. Shannon A, Alkhouri N, Carter-Kent C, et al. Ultrasonographic quantitative estimation of hepatic steatosis in children with NAFLD. J Pediatr Gastroenterol Nutr 2011; 53: 190-195.

25. Fitzpatrick E, Dhawan A. Noninvasive biomarkers in non-alcoholic fatty liver disease: current status and a glimpse of the future. World J Gastroenterol 2014; 20: 10851-10863.

26. Charatcharoenwitthaya P, Lindor KD. Role of radiologic modalities in the management of nonalcoholic steatohepatitis. Clin Liver Dis 2007; 11: 3754 .

27. Li Q, Dhyani M, Grajo JR, Sirlin C, Samir AE. Current status of imaging in non-alcoholic fatty liver disease. World J Hepatol 2018; 10: 530-542.

28. Guzmán-Aroca F, Frutos-Bernal MD, Bas A, et al. Detection of non-alcoholic steatohepatitis in patients with morbid obesity before bariatric surgery: preliminary evaluation with acoustic radiation force impulse imaging. Eur Radiol 2012; 22: 2525-2532.

29. Kim JR, Suh CH, Yoon HM, Lee JS, Cho YA, Jung AY. The diagnostic performance of shear-wave elastography for liver fibrosis in children and adolescents: a systematic review and diagnostic meta-analysis. Eur Radiol 2018; 28: 1175-1186.

30. Kamble R, Sodhi KS, Thapa BR, et al. Liver acoustic radiation force impulse (ARFI) in childhood obesity: comparison and correlation with biochemical markers. J Ultrasound 2016; 20: 33-42.
31. Marginean CO, Marginean C. Elastographic assessment of liver fibrosis in children: a prospective single center experience. Eur J Radiol 2012; 81: e870-e874.

32. Bailey SS, Youssfi M, Patel M, Hu HH, Shaibi GQ, Towbin RB. Shear-wave ultrasound elastography of the liver in normal-weight and obese children. Acta Radiol 2017; 58: 1511-1518.

33. Vajro P, Maddaluno S, Veropalumbo C. Persistent hypertransaminasemia in asymptomatic children: a stepwise approach. World J Gastroenterol 2013; 19: 2740-2751.

34. Mofrad P, Contos MJ, Haque M, et al. Clinical and histologic spectrum of nonalcoholic fatty liver disease associated with normal ALT values. Hepatology 2003; 37: 1286-1292.

35. Schwimmer JB, Dunn W, Norman GJ, et al. SAFETY study: alanine aminotransferase cutoff values are set too high for reliable detection of pediatric chronic liver disease. Gastroenterology 2010; 138: 1357-1364.

36. Wong VW, Vergniol J, Wong GL, et al. Diagnosis of fibrosis and cirrhosis using liver stiffness measurement in nonalcoholic fatty liver disease. Hepatology 2010; 51: 454-462.

37. Engelmann G, Gebhardt C, Wenning D, et al. Feasibility study and control values of transient elastography in healthy children. Eur J Pediatr 2012; 171: 353-360.

38. Hanquinet S, Rougemont AL, Courvoisier D, et al. Acoustic radiation force impulse (ARFI) elastography for the noninvasive diagnosis of liver fibrosis in children. Pediatr Radiol 2013; 43: 545-551. 